\title{
Wavelength-diversity transmission for fading mitigation in the atmospheric optical communication channel
}

\author{
Dirk Giggenbach $^{\mathrm{a}}$, Brandon L. Wilkerson ${ }^{\mathrm{b}}$, Hennes Henniger ${ }^{\mathrm{a}}$, Nicolas Perlot ${ }^{\mathrm{a}}$ \\ ${ }^{\mathrm{a}}$ German Aerospace Center (DLR), Institute of Communications and Navigation, \\ Oberpfaffenhofen, Germany \\ ${ }^{\mathrm{b}}$ US Air Force, Exchange Engineer at DLR
}

\begin{abstract}
Mitigation of index of refraction turbulence (IRT) effects is crucial in long-range atmospheric communication links. Diversity-transmission is one favorable way for fading compensation. One of several different diversity concepts is the exploitation of the wavelength-dependent index of refraction of the atmosphere, which leads to ideal stochastically independent fading at different wavelengths, depending on the scenario. This concept is here named wavelengthdiversity (WLD). Theoretical analysis and numerical simulation for the strength of this effect are given and verified by experimental tests.
\end{abstract}

Keywords: atmospheric optical free-space communication, wavelength-diversity transmission, atmospheric turbulence, atmospheric refraction gradient, atmospheric speckle pattern, diversity receiver

\section{INTRODUCTION}

Free-space optical communications systems in the atmosphere are heavily affected by signal fading which is caused by index-of-refraction turbulence (IRT). This fading is the result of turbulence-induced phase-perturbations which evolve in far-field speckle patterns and distorted wavefronts. These field perturbations are stochastic in space and time [8]. The resulting signal fades and surges can exceed $+/-20 \mathrm{~dB}$ in extreme cases, depending on link distance and path, and so can impair reliable data transmission. The fading-timescale is around several milliseconds in fixed applications and reduces with carrier velocity in mobile applications.

To broaden the applicability of reliable optical communications, the usage of fade-mitigation techniques is essential. While the simplest way to reduce intensity-fading is the enlargement of receiver-structures (as the correlation length of the intensity in typical scenarios is limited to some 10 centimeters), such measures are usually not applicable in mobile applications as smallest terminals are necessary there. Besides repeated data transmission and channel coding techniques (which imply delays on the order of several fade durations [10]), the only other way of reducing the overall variation of the received signal is transmission of the same modulated data simultaneously at different wavelengths.

Since the perturbing influence of the atmosphere on the transmitted electromagnetic waves is dependent on the wavelength, the speckle patterns caused by IRT have different distributions for different wavelengths. One reason for this is the index of refraction gradient of the earth's atmosphere with varying altitude. This causes the beams of different wavelengths to take slightly different paths and thus pass different turbulence volumes (see Fig. 1). Another reason is that the size of the speckle pattern itself is wavelength-dependent, which will lead to different patterns in long range (strong turbulence) links.

When two or more wavelengths of sufficient spectral distance are used for transmission through the same terminal apertures and the scenario has a sufficient diversity effect, the received powers will show statistically independent behavior for each channel (wavelength) and thus a classical diversity receiver scheme (selection combining receiver, equal-gain combining, or maximum-ratio combining) can be applied. Further, channels with statistically independent fading can be beneficially used by error correction coding schemes.

Contact: dirk.giggenbach@dlr.de; phone 49-8153-282821

Copyright 2006 Society of Photo-Optical Instrumentation Engineers. This paper was published in Proceedings of the SPIE Vol. 6304 and is made available as an electronic reprint with permission of SPIE. One print or electronic copy may be made for personal use only. Systematic or multiple reproduction, distribution to multiple locations via electronic or other means, duplication of any material in this paper for a fee or for commercial purposes, or modification of the content of the paper are prohibited. 
As the effect requires a large difference in the IoR of the air, one is usually limited to two wavelengths, one in the silicon domain around $800 \mathrm{~nm}$ and the other at around $1550 \mathrm{~nm}$ for InGaAs receivers (Fig. 2). Here we will show results of theoretical evaluation and practical testing of WLD for the two wavelengths $840 \mathrm{~nm}$ and $1550 \mathrm{~nm}$ with direct detection receivers. The use of wavelength diversity has been largely ignored in the past due to the unlikely nature of it being advantageous in conventional (short range) link scenarios. With mobile free-space optics communications (MFSO) becoming an important research topic for inter-aircraft and inter-HAP communications, there are new scenarios currently evolving with long horizontal paths which will benefit greatly from wavelength diversity [9].

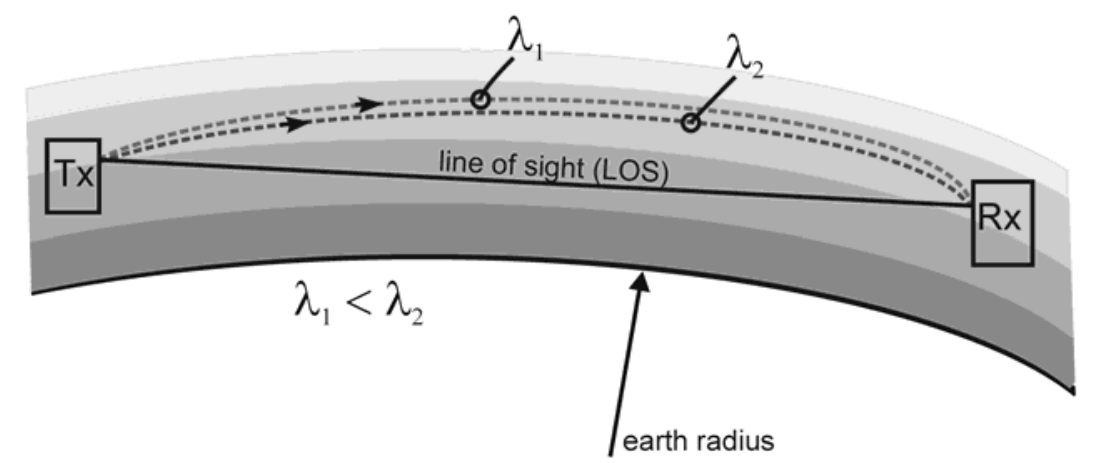

Fig. 1. Beam bending for different wavelengths passing through the atmospheric IoR-gradient which decreases exponentially with atmospheric altitude.

\section{THEORY OF WAVELENGTH-DIVERSITY TRANSMISSION}

As the WLD effect is based on the interplay of two physical principles as already mentioned above, and the estimation of near-ground IRT-strength is quite uncertain, an accurate quantitative assessment of the WLD-effect is difficult.

The diversity effect increases strongly with distance and turbulence strength when multiple scattering (strong turbulence) starts to govern the scenario. Also, of course, the increased separation of the two beams in the middle of the link causes stronger diversity. The separation of the two beams in the middle of the path even behaves more than proportionally to the path length, as shown in table 2 .

We start here with an analytical estimation of the vertical separation of two optical rays with different wavelengths, which both span from the Tx- to the Rx-aperture. Note that the divergence of both Tx-beams will of course be much larger than the angular value given by this separation. In Fig. 2, the index of refraction (IoR) over wavelength is given for different altitudes. Obviously, the difference in IoR is small at higher altitudes, but this is compensated by the much longer link distances typical for scenarios in the upper atmosphere (like inter-aircraft links or OIPLs).

Table 1. Index of refraction at $840 \mathrm{~nm}$ and $1550 \mathrm{~nm}$ at different altitudes

\begin{tabular}{|l|c|c|c|c|}
\cline { 2 - 5 } \multicolumn{1}{l|}{} & sea level & $3 \mathrm{~km}$ altitude & $10 \mathrm{~km}$ altitude & $25 \mathrm{~km}$ altitude \\
\hline IoR-1 at $840 \mathrm{~nm}$ & $2.702 \mathrm{E}-4$ & $1.996 \mathrm{E}-4$ & $9.582 \mathrm{E}-5$ & $1.394 \mathrm{E}-5$ \\
\hline IoR-1 at $1550 \mathrm{~nm}$ & $2.682 \mathrm{E}-4$ & $1.981 \mathrm{E}-4$ & $9.510 \mathrm{E}-5$ & $1.384 \mathrm{E}-5$ \\
\hline IoR(840) - IoR(1550) & $2.013 \mathrm{E}-6$ & $1.487 \mathrm{E}-6$ & $7.137 \mathrm{E}-7$ & $1.038 \mathrm{E}-7$ \\
\hline
\end{tabular}




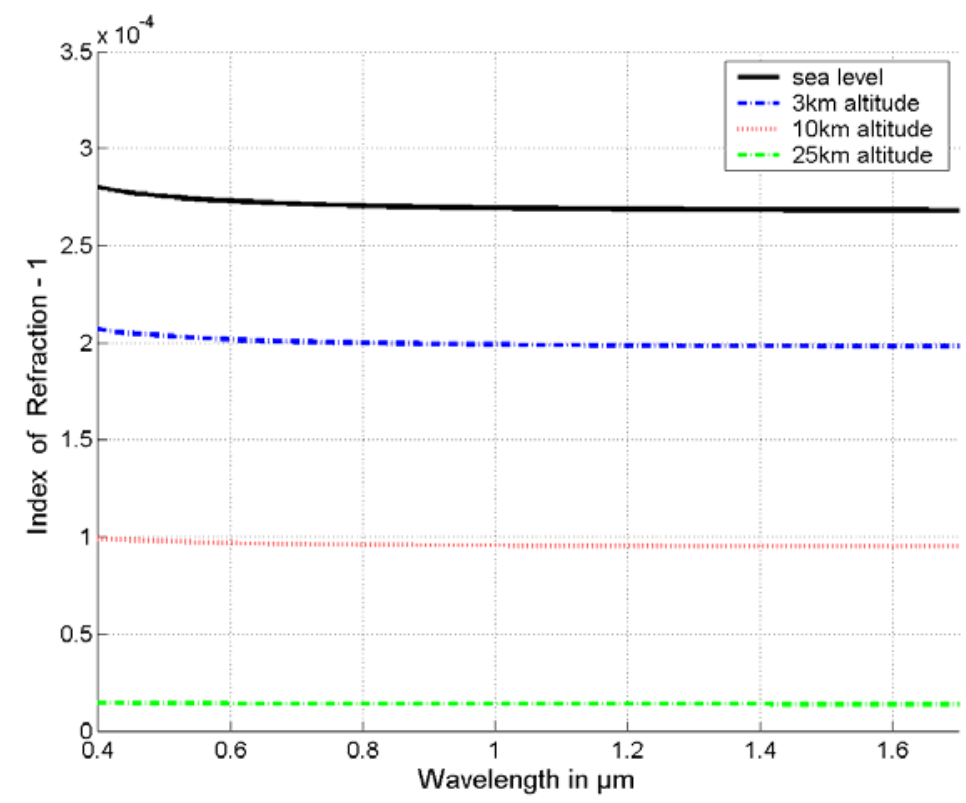

Fig. 2. Index of refraction over wavelength for sea level, $3 \mathrm{~km}, 10 \mathrm{~km}$, and $25 \mathrm{~km}$ altitude

If the model of standard exponential atmosphere [1] is used, the beam path can be calculated depending on the wavelength. This model can not be accurately applied in the boundary layer because of atmospheric interference, due to the temperature exchange with the ground. Nevertheless it can be used for the determination of the wavelength dependent vertical path separations for higher altitudes.

In this discussion, the curvature of the earth is unaccounted for. A z-y-Cartesian coordinate system is used where $y$ is the height above sea level and $z$ the distance along the ground.

The propagation of the optical beam is assumed to be almost parallel to the direction of the z-axis, which means that an infinitesimal distance of propagation $d s$ is nearly equal to the distance of the ground track $d z$. With these assumptions, the general wave equation can be written as:

$$
\frac{d}{d z}\left(\frac{d y}{d z}\right)=\frac{1}{n} \cdot \frac{d n}{d y}
$$

where $n(y)$ is the index of refraction, which decreases exponentially with the height $y$ above sea level, according to the standard exponential atmosphere model. Using $H$ as a scale height with a value of $7400 \mathrm{~m}$ and the differential index of refraction

$$
n^{\prime}(\lambda)=2.879 \cdot 10^{-4}+2.165 \cdot 10^{-18} m^{2} \cdot \lambda^{-2}
$$

the height dependent index of refraction $n(y)$ can be written according to the standard exponential atmosphere model from [2]:

$$
n(y)=1+n^{\prime} \cdot \exp (-y / H)
$$

With the assumption that $n^{\prime} \cdot \exp (-y / H)<<1$, the general wave equation can be written as a special wave equation: 


$$
\frac{d}{d z}\left(\frac{d y}{d z}\right)=-\frac{n^{\prime}(\lambda)}{H} \cdot \exp (-y / H)
$$

The integration of the special wave equation over $z$ gives the path equation $y(z)$ :

$$
y(z)=H \cdot \ln \left[-\frac{n^{\prime}(\lambda)}{H^{2}}\left(\frac{z^{2}}{2}+C_{1} \cdot z+C_{2}\right)\right]
$$

The integration constants in the path equation can be determined using the following boundary conditions:

Beam propagation starts at height $h_{0}$, which means $y(z=0)=h_{0}$. Therefore $C_{2}$ is fixed to:

$$
C_{2}=-\frac{H^{2}}{n^{\prime}} \cdot \exp \left(h_{0} / H\right)
$$

The second boundary condition is that the beam starts with an elevation angle of $\theta_{0}$, which means $\left.\frac{d y}{d z}\right|_{\substack{z=0 \\ y=h_{0}}}=\theta_{0}$. Now also $C_{l}$ is fixed to:

$$
C_{1}=-\frac{-\theta_{0} H}{n^{\prime}} \cdot \exp \left(h_{0} / H\right)
$$

If light paths start at the same point and meet a point-receiver, the start-beam elevation angle $\theta_{0}$ can be estimated using the assumption that the maximum beam height above sea level is approximately at the half link distance $L$ : $y_{\max }=y\left(z=\frac{L}{2}\right)$ (note: the function $y=\ln ($.$) is not symmetric to L / 2$ ). Additionally, the path equation must have a maximum in order to achieve a bending of the path to the ground.

With $\frac{d y}{d z}=0$ and $z=\frac{L}{2}$ the start beam elevation angle is approximately

$$
\theta_{0}=\frac{L}{2} \cdot \frac{n^{\prime}}{H} \cdot \exp \left(-h_{0} / H\right)
$$

As an example, the wavelength dependent paths for $840 \mathrm{~nm}$ and $1550 \mathrm{~nm}$ are calculated over a $13.4 \mathrm{~km}$ long path at height $h_{0}=570 \mathrm{~m}$ using the path equation. These parameters are close to those of test path \# 2 (see Table 4). Two beams starting at the same point and arriving at a point-receiver are considered, which means that the starting elevation angle for both paths is slightly different due to the wavelength dependent beam bending. Based on calculations, the maximum vertical path separation is about $0.6 \mathrm{~cm}$. This value matches the value calculated with the equation given in [3]. Plots of the optical paths and beam separation are shown in Fig. 3. 

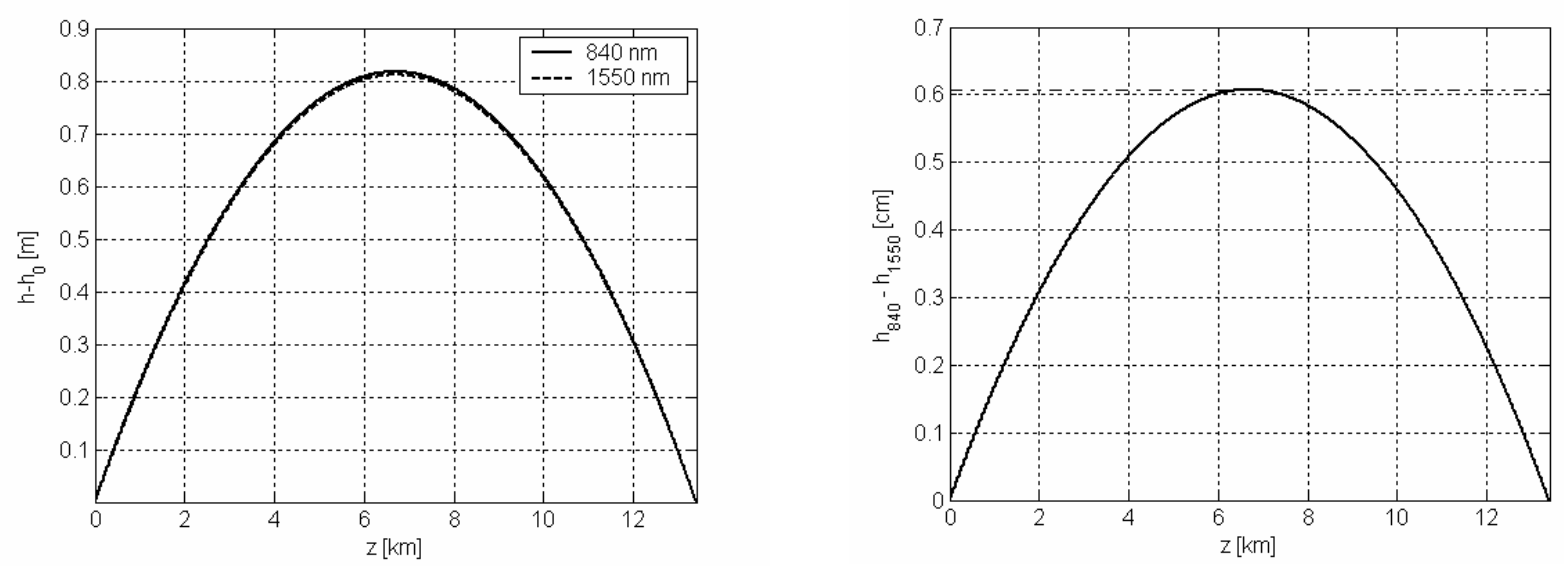

Fig. 3. Left: Optical paths of two beams with the same start- and arrival-point calculated from the path equation (5). Right: Vertical beam-path separation between the two beams. Wavelengths are $840 \mathrm{~nm}$ and $1550 \mathrm{~nm}$, altitude is $570 \mathrm{~m}$.

\section{NUMERICAL SIMULATION OF WLD-PERFORMANCE}

Simulations have been performed according to the split-step approach where turbulence is modelled by random phase screens [4], [5]. Two beams with wavelengths $840 \mathrm{~nm}$ and $1550 \mathrm{~nm}$ have been transmitted through the same phase screens. For the propagation of the second beam, however, the phase screens were shifted vertically (orthogonally to the beam direction) to account for the beam displacement due to the refraction layering [11].

\subsection{Simulated scenarios}

It has been assumed that the $\mathrm{C}_{\mathrm{n}}^{2}$ parameter has the fall-off of the Hufnagel-Valley model in the boundary layer. That is, $\mathrm{C}_{\mathrm{n}}^{2}$ has the following dependence on the height $h$ above the ground [6]

$$
C_{n}^{2}(h)=C_{n, 0}^{2} \exp \left(-\frac{h}{100 \mathrm{~m}}\right) \quad h \leq 1 \mathrm{~km}
$$

where $C_{n, 0}^{2}$ is the $C_{n}^{2}$ value at the ground. Inner and outer scales of turbulence have been fixed to $l_{0}=1 \mathrm{~cm}$ and $L_{0}=5 \mathrm{~m}$. The beam of each wavelength is collimated at the transmitter such that the divergence in the far field is $1 \mathrm{mrad}$ (full width half maximum). For the three test scenarios, Table 2 gives the values of $C_{n, 0}^{2}$ and $\Delta_{\max }$ which is the maximum separation distance between the two beams:

$$
\Delta_{\max }=h_{840}(z)-\left.h_{1550}(z)\right|_{z=L / 2} .
$$

The wave field was sampled as a $1024 \times 1024$ matrix with a sample interval of $d x \sim 5 \mathrm{~mm}$. For each scenario, 30 realizations have been run.

Table 2. Values of $\Delta_{\max }$ and $C_{n, 0}^{2}$ for the three test scenarios

\begin{tabular}{|l|c|c|}
\cline { 2 - 3 } \multicolumn{1}{c|}{} & $\Delta_{\max }$ & $C_{n, 0}{ }^{2}\left(C_{n}{ }^{2}\right.$ at ground $)$ \\
\hline Path 1 $(4.3 \mathrm{~km}$, day $)$ & $0.63 \mathrm{~mm}$ & $1 \times 10^{-13} \mathrm{~m}^{-2 / 3}$ \\
\hline Path 2 $(13 \mathrm{~km}$, night $)$ & $6.1 \mathrm{~mm}$ & $1 \times 10^{-14} \mathrm{~m}^{-2 / 3}$ \\
\hline Path 3 $(41 \mathrm{~km}$, day $)$ & $56 \mathrm{~mm}$ & $1 \times 10^{-13} \mathrm{~m}^{-2 / 3}$ \\
\hline
\end{tabular}




\subsection{Simulation results}

The intensity fields resulting from the simulations are assumed stationary. To evaluate the correlation between the intensity fields of each wavelength at the receiver, we consider the normalized cross-covariance defined as

$$
c_{\lambda_{1}, \lambda_{2}}(x, y)=\frac{\left\langle I_{\lambda_{1}}\left(x_{0}, y_{0}\right) I_{\lambda_{2}}\left(x_{0}+x, y_{0}+y\right)\right\rangle-\left\langle I_{\lambda_{1}}\right\rangle\left\langle I_{\lambda_{2}}\right\rangle}{\sqrt{\left\langle I_{\lambda_{1}}^{2}\right\rangle\left\langle I_{\lambda_{2}}^{2}\right\rangle}-\left\langle I_{\lambda_{1}}\right\rangle\left\langle I_{\lambda_{2}}\right\rangle}
$$

where the angular brackets denote ensemble averaging. In Fig. 4 to Fig. 6 , the cross-covariance $c_{\lambda_{1}, \lambda_{2}}(x, y)$ of the intensity fields corresponding to each of the two wavelengths is shown on the left. The same grey scale has been used for the three scenarios. As the path distance increases, the correlation spot becomes larger. In the scenario \#3, the two beams have propagated through much turbulence and their correlation at a given position is weaker than for the two other scenarios. As the path distance increases, the maximum distance $\Delta_{\max }$ increases. When $\Delta_{\max }$ is large enough, an offset of the correlation spot in the $y$ direction can be observed. This vertical offset is more visible for the scenario \#3.

On the right, distributions of the received powers normalized to their means are shown. This power is the power collected by an aperture of 5-cm diameter. The equal gain combining of both signals leads to a scintillation index close to that of the most favourable wavelength. The simulation results are summarized in Table 3.
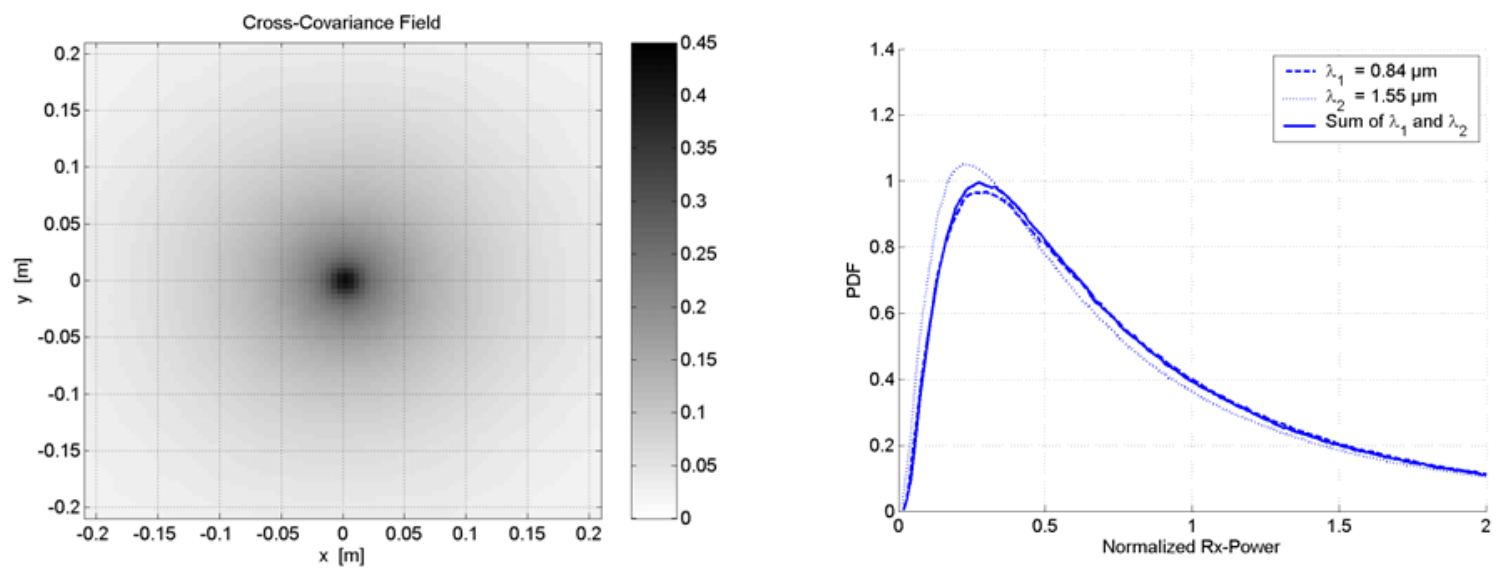

Fig. 4. Simulation results for test scenario \#1. Left: Cross-covariance of the intensity fields. Right: PDFs of the optical power received over a 5 -cm aperture.
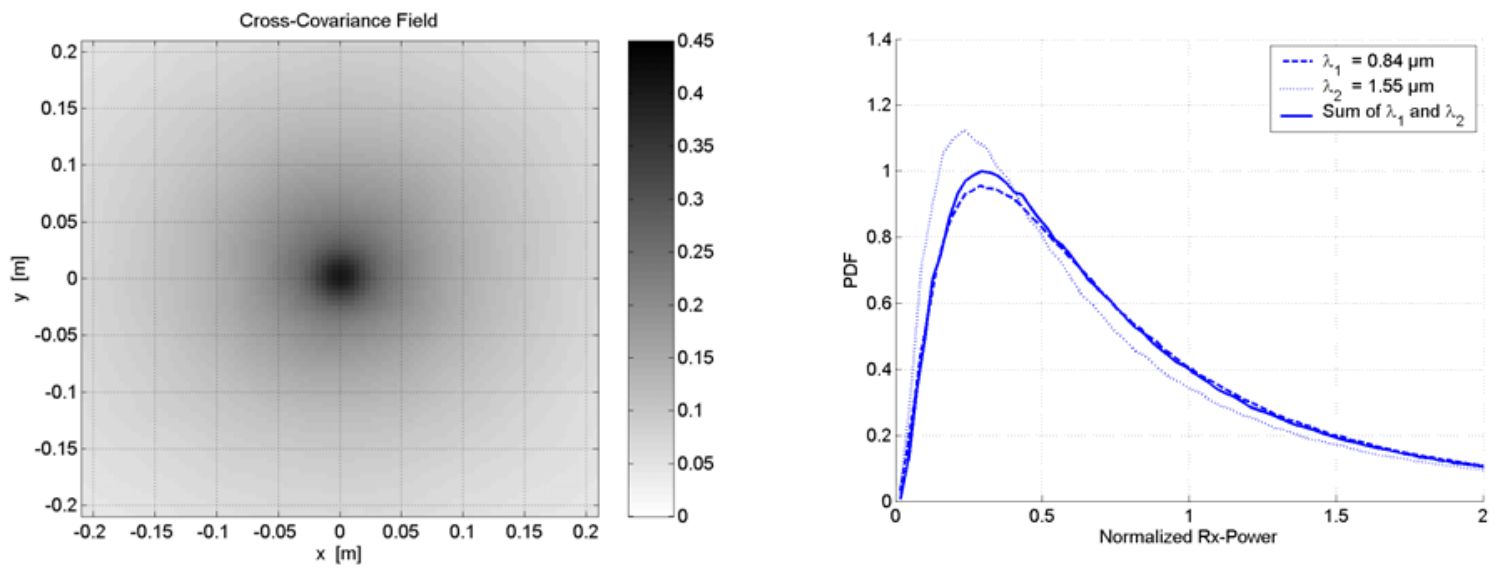

Fig. 5. Simulation results for test scenario \#2. Left: Cross-covariance of the intensity fields. Right: PDFs of the optical power received over a 5 -cm aperture. 

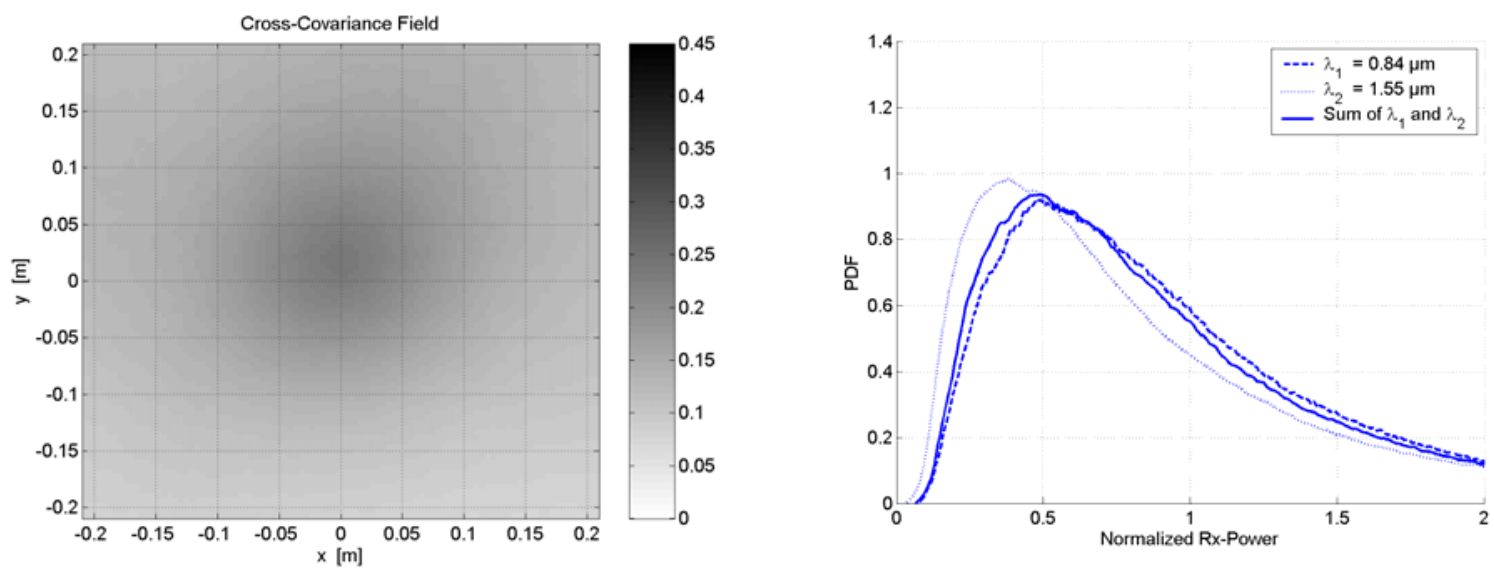

Fig. 6. Simulation results for test scenario \#3. Left: Cross-covariance of the intensity fields. Right: PDFs of the optical power received over a 5 -cm aperture.

Table 3. Simulation results for the 3 different test scenarios

\begin{tabular}{|c|c|c|c|c|c|c|}
\cline { 2 - 7 } \multicolumn{1}{c|}{} & \multicolumn{4}{c|}{ Intensity $I$} & \multicolumn{3}{c|}{ Power $P$} \\
\cline { 2 - 7 } & $\begin{array}{c}\sigma_{I}^{2} \text { at } \\
840 \mathrm{~nm}\end{array}$ & $\begin{array}{c}\sigma_{I}^{2} \text { at } \\
1550 \mathrm{~nm}\end{array}$ & $\begin{array}{c}c_{\lambda_{1}, \lambda_{2}}(x=0, y=0) \\
\text { Normalized Covariance }\end{array}$ & $\begin{array}{c}\sigma_{P}^{2} \text { at } \\
840 \mathrm{~nm}\end{array}$ & $\begin{array}{c}\sigma_{P}^{2} \text { at } \\
1550 \mathrm{~nm}\end{array}$ & $\begin{array}{c}\sigma_{P}{ }^{2} \text { of } \\
\text { the } \\
\text { sum }\end{array}$ \\
\hline $\begin{array}{c}\text { Path 1 } \\
(4.3 \mathrm{~km}, \text { day })\end{array}$ & 3.85 & 3.97 & 0.41 & 1.06 & 1.36 & 1.11 \\
\hline $\begin{array}{c}\text { Path 2 } \\
(13 \mathrm{~km}, \text { night })\end{array}$ & 3.25 & 3.57 & 0.36 & 1.15 & 1.76 & 1.27 \\
\hline $\begin{array}{c}\text { Path 3 } \\
(41 \mathrm{~km}, \text { day })\end{array}$ & 1.89 & 2.66 & 0.23 & 0.48 & 0.94 & 0.61 \\
\hline
\end{tabular}

\section{FREE-SPACE TESTING OF WLD-PERFORMANCE}

In July 2006 three trials were performed with three different link paths as shown in Fig. 7. While path 1 was too short to show good diversity reception (and mainly served for instrument calibration), the link budget at path 3 turned out to be too noisy due to daytime background light and offset-drifts were too fast and too strong. Therefore, night measurements at path 2 delivered the best results, with high SNR and negligible offset-drifts. 


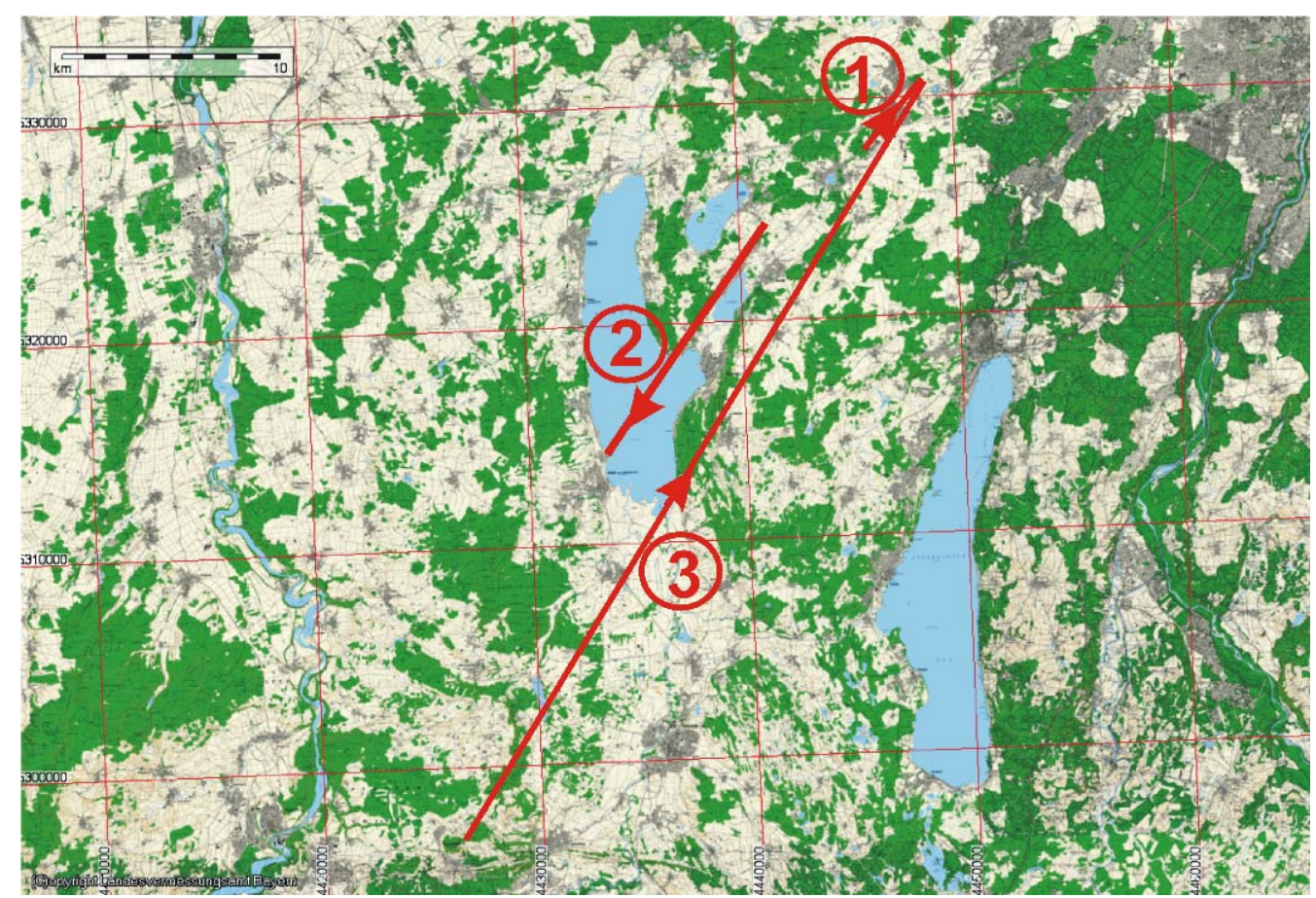

Fig. 7. Map of the three link scenarios for WLD trials

Table 4. Properties of the three link paths

\begin{tabular}{|l|c|c|c|c|}
\cline { 2 - 4 } \multicolumn{1}{c|}{} & Distance & Tx above sea level & Rx above sea level & $\begin{array}{c}\text { Time of } \\
\text { Measurement }\end{array}$ \\
\hline $\begin{array}{l}\text { Path 1 } \\
\text { OGS-OP to Gilching }\end{array}$ & $4.3 \mathrm{~km}$ & $590 \mathrm{~m}$ & $595 \mathrm{~m}$ & $\begin{array}{c}\text { afternoon, } \\
\text { bright sunlight }\end{array}$ \\
\hline $\begin{array}{l}\text { Path 2 } \\
\text { Meiling to St. Alban }\end{array}$ & $13.4 \mathrm{~km}$ & $590 \mathrm{~m}$ & $535 \mathrm{~m}$ & $\begin{array}{c}\text { night } \\
\text { completely dark }\end{array}$ \\
\hline $\begin{array}{l}\text { Path 3 } \\
\text { Peißenberg to Gilching }\end{array}$ & $41 \mathrm{~km}$ & $990 \mathrm{~m}$ & $595 \mathrm{~m}$ & $\begin{array}{c}\text { afternoon, } \\
\text { bright sunlight }\end{array}$ \\
\hline
\end{tabular}

\subsection{Test setup}

A $1550 \mathrm{~nm}$ fiber laser and a single mode fiber coupled $840 \mathrm{~nm}$ laser diode were used as transmitter signal sources. The 1550nm source was an IPG Photonics Erbium Fiber Laser (Model ELD-1-1550) and the 840nm source was a Lumics GmbH laser diode (LU0845M200) with a thermoelectric cooler controller (TEC-AI-5V-2.5V) and a Spectra Diode Labs laser diode driver (SDL-800M) Both lasers were combined using a Plank Optoelectronics wavelength division multiplexer (WD1L2BXXA133). Although this multiplexer is designed for $1550 / 980 \mathrm{~nm}$, it showed good performance when used with $840 \mathrm{~nm}$ as the second wavelength.

The realization of the receiver optics with of-the-shelf components proved to be most complicated and a first setup based on standard lenses, beam splitter cubes, and PIN-receivers had to be discarded as too insensitive. In a revised design, a special fiber-based chromatic beam-splitter was custom built by OECA-GmbH, which separated the two received wavelengths and focused the $840 / 1550 \mathrm{~nm}$ signals respectively on Si- and InGaAs-APDs. The receiver transimpedanceamplifier was most sensitive with a transimpedance of $1 \mathrm{M} \Omega$. An additional active filter with $2 \mathrm{kHz}$ bandwidth was integrated, all amplifier stages were DC-coupled. The voltage was then monitored by a storage oscilloscope. The setup is depicted in Fig. 8 and a summary of the setup data is given in Table 5. 

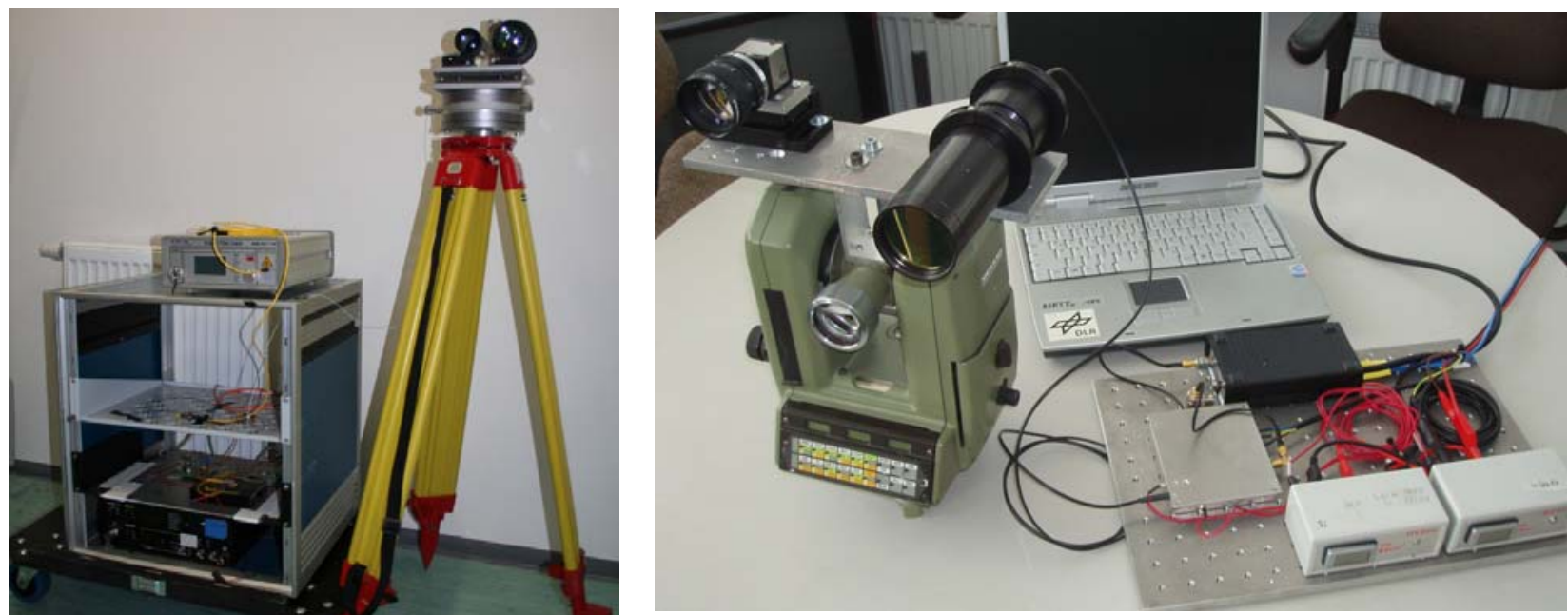

Fig. 8. Left: Transmitter setup showing the $840 \mathrm{~nm}$ and $1550 \mathrm{~nm}$ lasers and the transmitter optics mounted on an alignment system. Right: Receiver setup showing the optics mounted on a theodolite and a plate which holds the receiver electronics, high voltage sources, and data recording system.

Table 5. Summary of Setup Data

\begin{tabular}{|l|c|c|c|c|l|}
\hline \multicolumn{1}{|c|}{ parameter } & unit & min & typical & max & \multicolumn{1}{c|}{ remark } \\
\hline Tx-power 840nm & $\mathrm{mW}$ & 150 & 180 & 200 & $\begin{array}{l}\text { Powers estimated based on } \\
\text { operating forward current from } \\
\text { the laser diode driver }\end{array}$ \\
\hline Tx-power 1550nm & $\mathrm{mW}$ & 200 & 700 & 1000 & \\
\hline divergence 840nm & $\mathrm{mrad}$ & & 0.75 & & \\
\hline divergence 1550nm & $\mathrm{mrad}$ & & 1.0 & & \\
\hline $\begin{array}{l}\text { Rx-telescope: } \\
\text { aperture / focal length / } \\
\text { Rx-fibercore-diameter / FoV }\end{array}$ & $\begin{array}{l}\mathrm{mm} / \mathrm{mm} / \\
\mathrm{mm} / \mathrm{mrad}\end{array}$ & & $\begin{array}{l}50 / 100 / 100 \\
/ 1\end{array}$ & & FoV: Field-of-View \\
\hline voltage Si-APD (840nm) & $\mathrm{V}$ & & 231 & & \\
\hline voltage InGaAs-APD (1550nm) & $\mathrm{V}$ & & 53.3 & & \\
\hline
\end{tabular}

Temperature drift of the DC-coupled RFE was a major issue for proper offset adjustment of the DC-coupled RFEs. To monitor the offset, periodic Tx-signal disruptions were introduced during the measurements.

\subsection{Testing results}

Typical observed Rx-signal fluctuations are shown in Fig. 9. The received power vectors of the two signals showed partly correlated and partly uncorrelated behavior. This mixed behavior most likely is generated by different distances of the perturbing turbulence cells from the receiver - the closer to the receiver the perturbations are caused the more both signals will behave the same. 

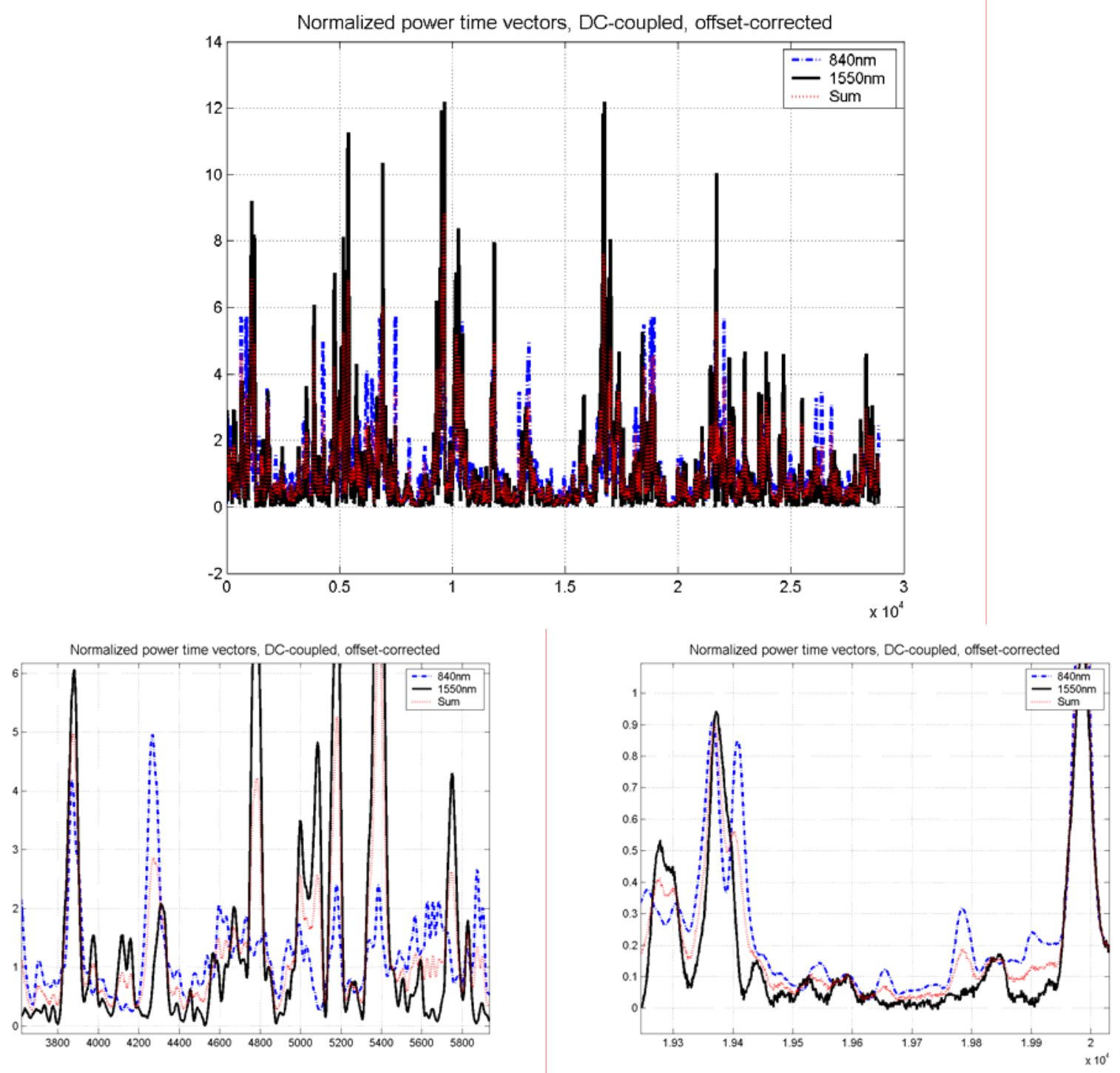

Fig. 9. Top: Typical Rx-power time signals of both wavelengths and their sum for path 2 (all signals normalized to a mean value of one). Bottom: Details of the top plot which show that there exist moments of correlated and uncorrelated behavior. Surges seem to be more correlated than fades. Solid line is for $1550 \mathrm{~nm}$-signal, chain-dotted line for $840 \mathrm{~nm}$ and dotted line for sum. One abscissa value equals $0.25 \mathrm{~ms}$

The PDFs of the signals (Fig. 10) somehow show a paradox behavior, as from simple theory one would expect less scintillation (accompanied by a more lognormal-like PDF) for the longer wavelength, while the shorter wavelength should have a more exponential-like behavior. Without going into detail of saturated scintillation, our experimental observation here is affirmed by the simulation results (Fig.5 and Fig. 6).

The cross-correlation coefficient as calculated for Table 6 is as a linear means dominated by the surges rather than by the fades. Therefore it is not a suitable parameter to estimate the WLD-benefit for communication systems, which are limited by the fading behavior. To give an impression of how communications could benefit from WLD we calculated the long term bit error rates of all signals $(840 \mathrm{~nm}$ alone, $1550 \mathrm{~nm}$ alone and the normalized sum, all three with the same mean Rx-power), using always the same receiver model based on the error-function with its argument behaving linear to the optical Rx-power. The operating point for the BER-curve was chosen as "BER $=10^{-9}$ with $\mathrm{P}_{\mathrm{Rx}}=1$ " and an additional $10 \mathrm{~dB}$ fading margin was introduced. The results are also given in Table 6. 

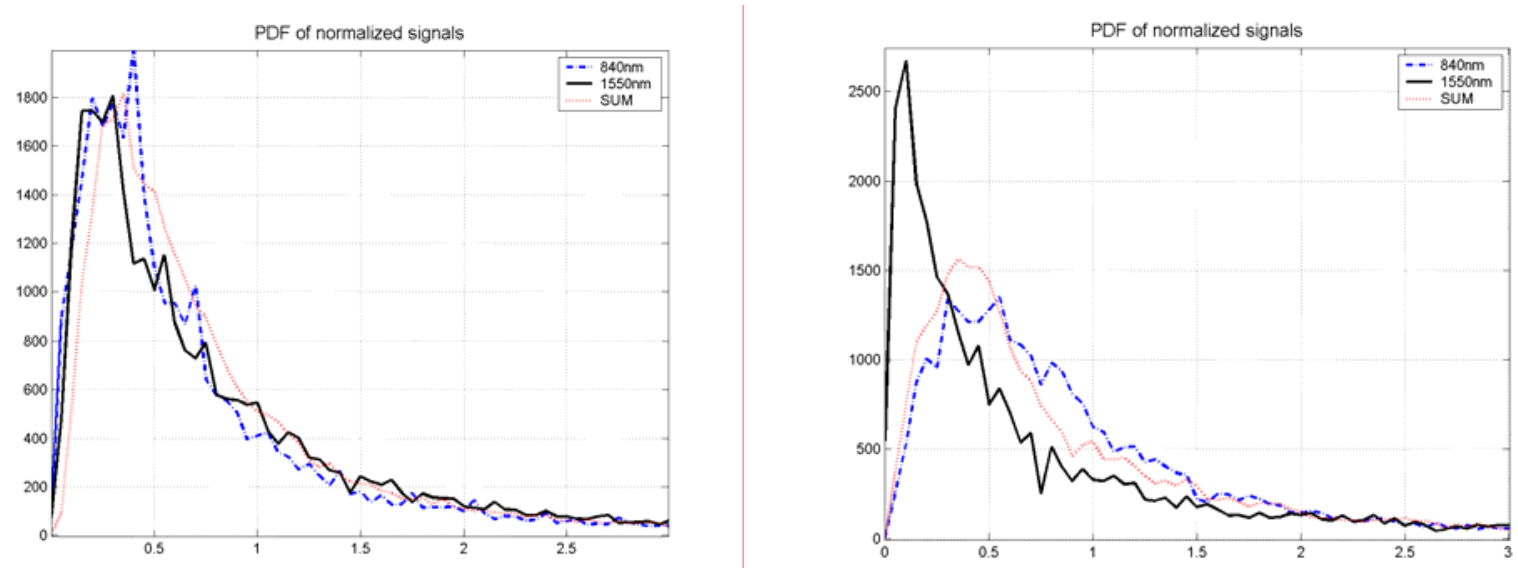

Fig. 10. Comparison of the probability density functions for path 1 (left) and path 2 (right). All power distributions normalized to a mean value of one.

Table 6. Summary of typical measurement values

\begin{tabular}{|l|c|c|c|}
\hline \multicolumn{1}{|c|}{ parameter } & Path 1 & Path 2 & Path 3 \\
\hline mean Rx-power 840nm & $\mathrm{x}$ & $5 \mathrm{nW}$ & $\mathrm{x}$ \\
\hline mean Rx-power 1550nm & $\mathrm{x}$ & $2 \mathrm{nW}$ & $\mathrm{x}$ \\
\hline Rytov-index for 840nm (calculated from theory) & 48.9 & 37.8 & 1085 \\
\hline Rytov-index for 1550nm (calculated from theory) & 23.9 & 18.5 & 531 \\
\hline power scint. index 840nm & 3.1 & 0.85 & $\mathrm{x}$ \\
\hline power scint. index 1550nm & 1.6 & 2.46 & $\mathrm{x}$ \\
\hline PSI of 840+1550 (with normalized mean powers) & 1.7 & 1.15 & $\mathrm{x}$ \\
\hline cross correlation coefficient of Rx-power & 0.50 & 0.44 & $\mathrm{x}$ \\
\hline calculated long term BER for 840nm signal & $7.0 \mathrm{E}-3$ & $1.9 \mathrm{E}-3$ & $\mathrm{x}$ \\
\hline calculated long term BER for 1550nm signal & $4.7 \mathrm{E}-3$ & $26 \mathrm{E}-3$ & $\mathrm{x}$ \\
\hline calculated long-term BER for normalized Sum & $1.0 \mathrm{E}-3$ & $2.9 \mathrm{E}-3$ & $\mathrm{x}$ \\
\hline
\end{tabular}

\section{CONCLUSIONS AND OUTLOOK}

The effect of wavelength diversity (WLD) was investigated theoretically, by numerical simulations, and by near-ground field trials with varying path lengths (from $4.3 \mathrm{~km}$ to $41 \mathrm{~km}$ ). The effect of WLD could be measured clearly in two out of three trial distances, while the measurements on the longest distance suffered from low SNR and thus no reliable stochastic evaluation could be performed with path \#3 data. Decorrelated signal behavior could clearly be observed on all three paths.

Communication quality improvements through WLD (estimated by long term BER) depend on the specific link scenario. The quality of the normalized equal gain combining receiver simulated here can also be lower than when using only the better signal (with the same total mean Rx-power). Selection combining or maximum ratio combining diversity receivers should perform much better here. The two paths tested successfully in this work certainly do not qualify for optimum WLD application. The distances were too short while the turbulence strength was too high, as already with the short path \#1 we observed PSIs over 3.

We would expect that much longer paths together with lower turbulence strength should benefit much more from this technology, making horizontal avionic links and Optical Inter-Platform Links (OIPLs) top candidates for the application of WLD transmission [7]. Especially with long OIPLs between HAPs at equal altitudes, we find the situation that the stronger turbulence will be encountered in the middle of the path where also the distance between the two wavelengths is at its maximum. This will cause very good decorrelation of both signals.

To fully exploit the benefits of WLD, custom-made optical components are necessary, like special lenses for the transmitter to allow near diffraction limited divergence at both wavelengths. As the IoR-increase rises towards shorter 
wavelength (Fig. 2), using lasers in the visible spectrum should provide an even stronger WLD effect. But the lack of laser sources for communications and the increased background light from the sun would be a drawback.

Future work on this topic should concentrate on the applicability of WLD in long-range scenarios at higher altitudes. As such trials would be connected with a high experimental effort, the scenarios should first be simulated thoroughly using numerical simulation tools. Also the concept of using more than two transmission wavelengths should be investigated.

\section{ACKNOWLEDGEMENTS}

The authors want to thank Joachim Horwath for his committed support during the trials.

The work presented in this paper was partly funded by the EU-FP6-project ATENAA (www.atenaa.org), which is aiming to establish broadband wireless optical communications for commercial aviation.

\section{ABBREVIATIONS}

$\begin{array}{ll}\text { HAP } & \text { High Altitude Platform } \\ \text { IoR } & \text { Index-of-Refraction } \\ \text { IRT } & \text { Index-of-Refraction Turbulence } \\ \text { MFSO } & \text { Mobile Free-Space Optics communications } \\ \text { OIPL } & \text { Optical Inter-Platform Links } \\ \text { PSI, } \sigma_{P}{ }^{2} & \left.\text { Power Scintillation Index; PSI }(\mathrm{s}(\mathrm{t}))=\left(<\mathrm{s}^{2}>/<\mathrm{s}\right\rangle^{2}\right)-1 \\ \text { Rx } & \text { Receiver } \\ \text { Tx } & \text { Transmitter } \\ \text { WLD } & \text { WaveLength Diversity }\end{array}$

\section{REFERENCES}

1. Van der Werf, "Ray Tracing and Refraction in the Modified US1976 Atmosphere", App. Opt. , Vol 42, Iss.3, pp354-366 (Jan. 2003)

2. Parsons, D. The mobile radio propagation channel. Pub. Pentech Press, London, 1992

3. R. Purvinskis, D. Giggenbach, H. Henniger, N. Perlot, F. David: Multiple Wavelength Free-Space Laser Communications. Free-Space Laser Communications Technologies XV, volume 4975 of Proc. of SPIE, 2003.

4. J.M. Martin, S.M. Flatté, "Intensity images and statistics from numerical simulation of wave propagation in 3-D random media", Applied Optics 27(11), S. 2111-2126, 1988

5. J. Horwath, N. Perlot, D. Giggenbach, R. Jüngling, "Numerical simulations of beam propagation through optical turbulence for high-altitude platform crosslinks," Proc. of SPIE 2004, Vol. 5338B (2004)

6. R. R. Beland, "Propagation through atmospheric optical turbulence", IR/EO Handbook, vol. 2, F. G. Smith, Ed. SPIE Press Bellingham (WA), pp. 157-232. (1993)

7. D. Giggenbach, R. Purvinskis, M. Werner, "Stratospheric optical inter-platform links for high altitude platforms", Proc. AIAA Conf. on Sat. Communications, Montreal, May 2002

8. L.C. Andrews, R.L. Philips, Laser Beam propagation through Random Media, SPIE Press, Bellingham (WA), 1998

9. N. Perlot, J. Horwath, D. Giggenbach, "Measurements of the Beam-Wave Fluctuations over a 142-km Atmospheric Path", SPIE - Optics \& Photonics 2006, 13-17 August 06

10. H. Henniger, "Packet-Layer Forward Error Correction Coding for Fading Mitigation", SPIE - Optics \& Photonics 2006, 13-17 August 06

11. N. Perlot, J. Horwath, R. Jüngling. "Simulated spatio-temporal realizations of optical-wave propagation through atmospheric turbulence", Proc. of SPIE 2005, Vol. 5712, Jan05 